\title{
Significance of Immune-related Genes in the Diagnosis and Subtype Classification of Childhood Asthma
}

\section{Bing Dai}

Department of Pediatric, Shengjing Hospital of China Medical University, Shenyang, China

\section{Feifei Sun}

Department of Ultrasound, Shengjing Hospital of China Medical University, Shenyang, China

\section{Xuxu Cai}

Department of Pediatric, Shengjing Hospital of China Medical University, Shenyang, China

\section{Chunlu Li}

Department of Pediatric, Shengjing Hospital of China Medical University, Shenyang, China

\section{Henan Liu}

Department of Ophthalmology, Shengjing Hospital of China Medical University, Shenyang, China

\section{Yunxiao Shang ( $\nabla$ sxy15088706914@163.com )}

Shengjing Hospital of China Medical University https://orcid.org/0000-0002-8885-4937

\section{Research}

Keywords: Childhood asthma, Immune-related genes, Random forest model, Nomogram, Consensus clustering, Immunocyte

Posted Date: September 23rd, 2020

DOl: https://doi.org/10.21203/rs.3.rs-76979/v1

License: (c) (i) This work is licensed under a Creative Commons Attribution 4.0 International License. Read Full License 


\section{Abstract}

Background Childhood asthma is one of the most common causes of hospitalization in children, causing huge economic losses worldwide. The immune system was closely linked to the occurrence and development of childhood asthma. The main purpose of this research is to explore the role of immunerelated genes in the occurrence and treatment of childhood asthma.

Methods GSE40732 dataset and GSE40888 dataset were respectively regarded as training dataset and texting dataset in this article. We performed weighted gene co-expression network analysis (WGCNA) to select immune-related genes associated with the occurrence of childhood asthma based on the training dataset. Random forest (RF) model was established to screen the optimal variables to predict the occurrence of childhood asthma among these significant immune-related genes. Childhood asthma patients were grouped by the consensus clustering method based on the optimal variables. The grouping results were validated in the texting dataset.

Results 69 significant immune-related genes associated with the occurrence of childhood asthma were screened through WGCNA. RF model indicated that 10 optimal variables among these significant immune-related genes can reasonably distinguish normal children and childhood asthma. Childhood asthma patients were classified into two molecular subtypes (Sub1 and Sub2) based on the 10 optimal variables using consensus clustering analysis. More interestingly, childhood asthma patients in Sub1 have higher inflammatory response than Sub2.

Conclusions Our research selected 10 significant immune-related genes related to the occurrence of childhood asthma. We classified childhood asthma patients into two molecular subtypes with different immune cell infiltration based on the 10 significant immune-related genes, which may provide the basis for individualized treatment.

\section{Background}

Bronchial asthma is the most common chronic respiratory disease in children. The recent increase in the incidence of childhood asthma not only threatens child health but also poses a significant public health issue in industrialized countries ${ }^{1}$. According to the international study of asthma and allergies in childhood (ISAAC), the prevalence of childhood asthma in some countries exceeds $10 \%^{2}$. Childhood asthma seriously undermines the quality of life and increases the medical burden on society ${ }^{3}$. Therefore, investigating the pathogenesis of childhood asthma and exploring precise treatment strategies for this disease have been deemed important.

Certain factors are known to play an important role in the pathogenesis of childhood asthma. Continuing exploration of immune mechanisms underlying childhood asthma as well as the role played by allergic factors in its pathogenesis has led to immunotherapy being developed as a new treatment line for childhood asthma. Immunotherapy is divided into non-specific immunotherapy and specific immunotherapy (SIT). Non-specific immunotherapy is associated with immune avoidance or using anti- 
allergic drugs. Specific immunotherapy refers to the process of identifying a patient's allergens via pathogenic detection, applying the corresponding allergen extracts to induce immune stimulation, and

gradually increasing the stimulation dosage until immune tolerance is achieved ${ }^{4-6}$. In recent years, several randomized, controlled trials have demonstrated the efficacy of SIT in treating asthma. Although randomized controlled trials have confirmed the clinical benefits of SIT, the risk of developing severe allergic reactions due to SIT persists ${ }^{7}$. Therefore, investigating immune mechanisms underlying childhood asthma and adopting specific, individualized immunotherapies have been considered vital.

The current study utilized weighted gene co-expression network analysis (WGCNA) to select immunerelated genes associated with childhood asthma. The Random Forest (RF) model was then used to screen 10 significant immune-related genes that could be potentially used to predict the occurrence of childhood asthma. In addition, consensus clustering analysis was performed to classify childhood asthma patients into two molecular subtypes based on these 10 significant immune-related genes, with the expectation that these subtypes may provide a basis for individualized treatment.

\section{Materials And Methods \\ Data acquisition}

The training and testing datasets, GSE40732 ${ }^{8}$ and GSE40888 ${ }^{9}$, were obtained from the Gene Expression Omnibus database (http://www.ncbi.nlm.nih.gov/geo/, RRID:SCR_007303). GSE40732 contained 97 nonasthmatic and 97 asthmatic patients, while GSE40888 dataset contained 40 non-asthmatic and 65 asthmatic patients. Moreover, 2499 immune-related genes were downloaded from the ImmPort database (https://immport.niaid.nih.gov, RRID:SCR_012804) ${ }^{10}$.

\section{Construction of the co-expression network}

The 'WGCNA' package in R software \https://labs.genetics.ucla.edu/horvath/CoexpressionNetwork/, RRID:SCR_003302『was used to establish the co-expression network. The first step was to construct the matrix, which consisted of three matrices (similarity, adjacency, and topological matrices) and two matrix transformations (similarity matrix converted into the adjacency matrix and adjacency matrix converted into the topological matrix). The similarity matrix was used to construct a network according to the degree of correlation between genes. The adjacency matrix defines the adjacency function of the WGCNA network. The topological matrix refers to the degree of difference between nodes. The similarity matrix was converted into the adjacency matrix according to the formula, amn $=\mathbb{} \mathrm{cmnn} \mathbb{\Xi}^{\mathrm{b}}$, where amn and $\mathrm{cmn}$ refer to the correlation and connection coefficients between genes $m$ and gene $n$, respectively, and $b$ represents soft threshold. The adjacency matrix was converted into the topological matrix using an appropriate soft threshold. The second step obtained the co-expression module through the dynamic shear tree. A co-expression module is interpreted as a collection of genes with high topological overlap similarities. A dynamic shear tree is an algorithm consisting of two parts, mapping and dynamic shear 
gene trees. Gene tree mapping calculates the co-expression correlation coefficient of each gene according to its expression level, clusters the genes, and maps the gene tree. Dynamic cutting refers to pruning the gene tree and fusing the pruned gene tree into multiple gene modules ${ }^{11-13}$. In the present study, we selected a gene module highly correlated with childhood asthma as the 'hub' module for further research. Next, we applied gene ontology (GO) analysis based on the genes in the 'hub' module using 'clusterProfiler' package in R software to explore the potential mechanisms underlying the function of these genes.

\section{Random forest model construction}

Random forest (RF) is a constituent supervised learning method, which can be considered an extension of a decision tree. Random forest builds prediction models by sampling objects and variables, generates multiple decision trees, and classifies objects in turn. Finally, the classification results of each decision tree are summarized. The mode category of all prediction categories is selected as the target category predicted by the random forest. Our study used the 'RandomForest' package in R software to establish a random forest model that enabled the selection of optimal variables that predict childhood asthma ${ }^{11}$.

\section{Nomogram model construction}

The nomogram model integrates multiple prediction indicators according to a certain proportion allocation and visualizes prediction results. To predict the prevalence of childhood asthma patients, we constructed a nomogram model based on selected significant genes using the ' $r m s$ ' package in $\mathrm{R}$ software. We estimated morbidity by adding the points of each variable to obtain total points. The calibration curve was used to evaluate the consistency of our predicted values against actual reality. Decision curve analysis (DCA) was performed, and a clinical impact curve was plotted to assess whether decisions based on the model were beneficial to the patient.

\section{Identification of molecular subtype}

Childhood asthma patients were grouped using the consensus clustering method based on the optimal variables. 'ConsensusClusterPlus' package in $\mathrm{R}$ software was used to perform consensus clustering. Consensus clustering is an algorithm that is used to identify each member and its subgroup number and verify clustering rationality based on resampling ${ }^{14}$.

\section{Estimation of the abundance of immune cells}

Single sample gene set enrichment analysis (SSGSEA) was used to evaluate the abundance of immune cells in non-asthmatic and asthmatic samples. First, ssGSEA was used to sequence the gene expression 
levels in the samples in order to obtain their rank. Next, we searched for these genes in the input data set, following which the expression levels of these genes were summed. Based on the above evaluation, we obtained the abundance of immune cells in each sample.

\section{Statistical analysis}

One-way ANOVA and Kruskal-Wallis tests were used to compare differences between groups. All parametric analyses were based on two-tailed tests, the statistical significance of which was set at $p<$ 0.05. All statistical analyses were performed using R 4.0.0.

\section{Results}

\section{Identification of significant immune-related genes associated with childhood asthma}

Sample quality was detected using the training set GSE40732 by the 'flash cluster' package in R software. The results indicated that there were no outlier samples in the data set (Fig. 1 A). Immune-related gene expression profile data in the training set were used to establish the co-expression network. First, we used the degree of correlation between immune-related genes to obtain the similarity matrix. Next, we converted the similarity matrix into an adjacency matrix, according to the formula, amn $=\llbracket c m n \llbracket b$. Finally, we converted the adjacency matrix into a topological matrix using an appropriate soft threshold $(b=4$; R2= 0.98; Figs. 1 B-E). Five gene modules were obtained via the dynamic shear tree (Fig. 2 A). Moduletrait relationships indicated that the yellow module showed the highest correlation with childhood asthma (Fig. 2 B; p=0.01). All 69 genes in the yellow module were selected as significant immune-related genes suitable for further research (Fig. $2 \mathrm{C}$ ). To explore the potential mechanisms underlying the role of significant immune-related genes in childhood asthma, GO analysis was performed using the 'clusterProfiler' package in R software. The results revealed that significant immune-related genes may participate in childhood asthma by inducing immune activation (Fig. 3).

\section{Random forest model construction}

The 69 significant immune-related genes were screened to determine the optimal variables capable of predicting the occurrence of childhood asthma, using the generalized linear (GLM), RF, and support vector machine (SVM) models, all of which were established separately using the 'DALEX' package. The RF model, which showed the least residual distribution, was selected as the best model to predict childhood asthma (Figs. 4 A, B). We evaluated the importance of significant immune-related genes in predicting the occurrence of childhood asthma based on the established RF model. We visualized the top 30 genes after ranking these genes according to their importance (Fig. 4 C), where '\%IncMSE' represents an increase in mean squared error. The more important a predictor gene is, the greater the prediction error will be, when 
its value is randomly replaced. 'IncNodePurity' represents the increase in node purity, which indicates the effect of each gene on the heterogeneity of observations at each node of the classification tree. A 10 -fold cross validation was performed to select genes. These results indicated that the predicted results were more stable and exhibited lower cross validation errors when the number of genes was in the top 10 . Selection of the top 10 genes was based on the 'IncNodePurity' values corresponding to these genes, and the 10 genes selected were CD244, DDX58, CMTM2, NRG1, HLA-DPA1, TNFSF13B, TNFSF13, CMKLR1, $P S M E 2$, and SEMA6B.

\section{Nomogram model construction}

A nomogram model based on the selected top 10 genes was constructed, using the 'rms' package in $R$, to predict the prevalence of childhood asthma patients (Fig. 5 A). Calibration curves revealed that the predictivity of the nomogram model was accurate (Fig. 5 B). The 'rmda' package was used to plot the DCA curve. The red line remained above the grey and black lines from 0.2 to 0.8 , indicating that decisions based on the nomogram model may benefit childhood asthma patients (Fig. $5 \mathrm{C}$ ). We further plotted the clinical impact curve to more intuitively assess the clinical impact of the nomogram model. The predicted number of high-risk patients was greater than that of high-risk patients per event, indicating that the predictive power of the nomogram model was remarkable. (Fig. 5 D).

\section{Identification of two molecular subtypes of childhood asthma}

Consensus clustering enables childhood asthma patients to be divided into two molecular subtypes based on the expression profiles of the top 10 genes selected from the training dataset. Matrix heat maps were clearly separated at $k=2$ (Fig. 6 A). The rows and columns of the matrix heat map represent samples. The values of the consistency matrix are colored white to dark blue from 0 (impossible to cluster together) to 1 (always clustered together). The cumulative distribution function (CDF) curve indicated that the CDF reaches a value that approximates the maximum value at $\mathrm{k}=2$ (Fig. $6 \mathrm{~B}$ ). The item cluster membership across different k numbers that tracks cluster history is presented (Fig. 6 C). Classification stability based on the selected top 10 genes was successfully verified using the testing dataset (Supplementary Fig. 1).

\section{Landscape of immune infiltration in childhood asthma}

SSGSEA was used to evaluate the abundance of 28 infiltrating immune cells in childhood asthma patients. Myeloid-derived suppressor cells (MDSCs) exhibited the highest infiltration abundance among these 28 immune cell types (Fig. 7 A; Supplementary Fig. 2 A). Differential analysis of the training dataset indicated that activated CD8 T, gamma delta T, T follicular helper, CD56bright natural killer, immature dendritic, and natural killer T cells displayed higher levels of infiltration abundance in asthma patients 
compared to those in non-asthma patients (Fig. 7 B). However, the abundance of activated CD8 T cell infiltration in asthma patients in the testing dataset was lower than that in non-asthma patients (Supplementary Fig. 2 A). The imbalance between the numbers of non-asthma and asthma patients in the testing dataset may lead to immune infiltration results that are different from those in the training dataset. Moreover, we explored relative immune cell infiltration levels in the following two molecular subtypes: Sub1 and Sub2. Childhood asthma patients in Sub1 displayed higher immune cell infiltration levels than those in Sub2 (Figs. 8 A \& B). The major histocompatibility class 1 (MHC1) complex is necessary to provide endogenous cellular antigens to circulating $T$ cells. In the training dataset, the expression levels of $H L A-D M B, H L A-D O A$, and $H L A-D O B$ in Sub1 were higher than those in Sub2 (Fig. $8 \mathrm{C}$ ). In the testing dataset, the expression levels of HLA-DRA, HLA-J, HLA-DPB1, HLA-DQB2, HLA-C, HLA-DMA, HLA-DPA1, HLA-DMB, HLA-DPA2, and HLA-DRB1 in Sub1 were higher than those in Sub2 (Fig. $8 \mathrm{D}$ ).

\section{Discussion}

Asthma, which is the most common chronic respiratory disease in children, causes serious harm to the physical and mental health of children ${ }^{15,16}$. Asthma causes chronic inflammation of the airways through various cellular components and cytokines. Its pathogenesis is extremely complex. The genes encoding these cytokines determine the occurrence and development of asthma ${ }^{17}$. Therefore, exploring immunological mechanisms underlying childhood asthma as well as identifying biological markers that predict asthma progression is vital for reducing the incidence of childhood asthma and implementing bilevel preventive measures.

'WGCNA' was used to screen 69 immune-related genes associated with childhood asthma to explore immunological mechanisms underlying this disease. $\mathrm{GO}$ analysis revealed that the potential mechanism underlying the function of these 69 genes may be associated with immune activation. RF model screened the top 10 genes, namely, CD244, DDX58, CMTM2, NRG1, HLA-DPA1, TNFSF13B, TNFSF13, CMKLR1, $P S M E 2$, and $S E M A 6 B$, and used these 10 genes to establish a nomogram model that predicts the prevalence of childhood asthma. The DCA curve indicated that decisions based on the nomogram model could benefit childhood asthma patients. Thus, we contend that these 10 genes are highly correlated with the occurrence and development of childhood asthma.

CD244 is a member of the signaling lymphocyte activation molecule (SLAM) family, which plays an important role in regulating the activity of NK cells, monocytes, $T$ cells, and eosinophils ${ }^{18}$. Gangwar et al. reported that CD244 mediates the activation of eosinophils and participates in the development of human asthma ${ }^{19}$. DExD/H-Box Helicase 58 (DDX58), also known as reticonic acid induced gene I (RIG-I), activates and regulates the expression of various inflammatory factors, thereby initiating innate and specific immune responses ${ }^{20}$. Many studies have shown that RIG-I plays an important role in the inflammatory response of asthma induced by viruses ${ }^{21-23}$. Human leukocyte antigen (HLA) is a gene complex closely related to human immune function. The results of a study conducted by Shi et al., indicating that HLA-DPA1 may participate in the inflammatory response of childhood asthma, were 
consistent with those of the current study ${ }^{24}$. TNFSF13B and TNFSF13, members of the tumor necrosis factor (TNF) family, are important B cell stimulators ${ }^{25}$. Kumar et al. revealed that the genetic variations in the TNF family were associated with the aggravation rate of asthma ${ }^{26}$. Chemokine like receptor 1 (CMKLR1), which is a G protein-coupled receptor, plays an important role in inflammatory and immune responses ${ }^{27}$. CMKLR1 reportedly relieved allergic airway inflammation of asthma patients ${ }^{28}$. CMTM2, which belongs to the CMTM family, is highly expressed in peripheral blood mononuclear cells and is associated with numerous autoimmune diseases ${ }^{29}$. Neureg-ulin-1 (NRG-1), which is a member of the NRG gene family, acts as a paracrine factor in dendritic cells and is involved in tumor immunity ${ }^{30}$. SEMA6B is associated with macrophages ${ }^{31}$. Proteasome activator complex subunit 2 (PSME2) functions in antigen presentation and lymphocyte activation ${ }^{32}$. To the best of our knowledge, to date, no studies have described the relationship between CMTM2, NRG-1, SEMA6B, and PSME2 and asthma.

Consensus clustering analysis classified childhood asthma patients into two molecular subtypes based on the top 10 genes. Childhood asthma patients in Sub1 displayed a higher inflammatory response than those in Sub2. We propose that patients in Sub1 may be more sensitive to a specific type of immunotherapy. Currently we are unable to verify our premise by data analysis due to a lack of specific immunotherapy-related datasets in the database. We believe that our classification of childhood asthma patients may enable individualized immunotherapy to be successfully implemented against childhood asthma patients.

\section{Conclusions}

In conclusion, the current study selected 10 immune-related genes associated with childhood asthma and established a nomogram model that predicts the prevalence of childhood asthma. Based on the top 10 genes, we further identified molecular subtypes that display differential inflammatory responses, which may enable the development of individualized immunotherapy for childhood asthma patients.

\section{Abbreviations}

WGCNA: weighted gene co-expression network analysis; RF: random forest; ISAAC: international study of asthma and allergies in childhood; SIT: specific immunotherapy; GO: gene ontology; DCA: decision curve analysis; SSGSEA: single sample gene set enrichment analysis; GLM: generalized linear; SVM: support vector machine

\section{Declarations}

\section{Ethics approval and consent to participate}

Not applicable 


\section{Consent for publication}

Not applicable

\section{Availability of data and materials}

All data generated or analysed during this study are included in this published article and its supplementary information files

\section{Competing interests}

The authors declare that they have no competing interests

\section{Funding}

Not applicable

\section{Authors' contributions}

Bing Dai, Feifei Sun, Xuxu Cai, Chunlu Li, Henan Liu, Yunxiao Shang conceived and designed the study. Bing Dai, Feifei Sun Yunxiao Shang developed the methodology. Bing Dai, Feifei Sun, Xuxu Cai, Yunxiao Shang analyzed and interpreted the data. Bing Dai, Henan Liu, Yunxiao Shang wrote, reviewed, and/or revised the manuscript.

\section{Acknowledgments}

We thank the authors who provided the GEO public datasets.

\section{References}

1. Lejeune S, Deschildre A, Rouzic OL, et al. Childhood asthma heterogeneity at the era of precision medicine: Modulating the immune response or the microbiota for the management of asthma attack. Biochemical pharmacology 2020; 179(114046.

2. Asher MI, Montefort S, Björkstén B, et al. Worldwide time trends in the prevalence of symptoms of asthma, allergic rhinoconjunctivitis, and eczema in childhood: Isaac phases one and three repeat multicountry cross-sectional surveys. Lancet. 2006;368(9537):733-43.

3. Ebmeier $S$, Thayabaran D, Braithwaite I, et al. Trends in international asthma mortality: Analysis of data from the who mortality database from 46 countries (1993-2012). Lancet (London, England) 
2017; 390(10098):935-945.

4. Dhami S, Kakourou A, Asamoah F, et al. Allergen immunotherapy for allergic asthma: A systematic review and meta-analysis. Allergy. 2017;72(12):1825-48.

5. Kanmaz HG, Harmanci K, Razi C, et al. Specific immunotherapy improves asthma related quality of life in childhood. Allergologia et immunopathologia. 2011;39(2):68-72.

6. PA Eggleston. Allergen-specific immunotherapy in childhood asthma. Curr Opin Pediatr. 1997;9(6):582-4.

7. Abramson MJ, Puy RM, Weiner JM. Injection allergen immunotherapy for asthma. The Cochrane database of systematic reviews 20108):Cd001186.

8. Yang IV, Pedersen BS, Liu A, et al. DNA methylation and childhood asthma in the inner city. J Allergy Clin Immunol. 2015;136(1):69-80.

9. Raedler D, Ballenberger N, Klucker E, et al. Identification of novel immune phenotypes for allergic and nonallergic childhood asthma. J Allergy Clin Immunol. 2015;135(1):81-91.

10. Bhattacharya S, Andorf S, Gomes L, et al. Immport: Disseminating data to the public for the future of immunology. Immunol Res. 2014;58(2-3):234-9.

11. Bao X, Shi R, Zhao T, Wang Y. Mast cell-based molecular subtypes and signature associated with clinical outcome in early-stage lung adenocarcinoma. Molecular oncology. 2020;14(5):917-32.

12. Wang $P$, Zheng $H$, Zhang J, et al. Identification of key gene modules and genes in colorectal cancer (crc) by co-expression analysis weighted gene co-expression network analysis (wgcna). Bioscience reports 2020.

13. Ai D, Wang Y, Li X, Pan H. Colorectal cancer prediction based on weighted gene co-expression network analysis and variational auto-encoder. Biomolecules 2020; 10(9).

14. Wilkerson MD, Hayes DN. Consensusclusterplus: A class discovery tool with confidence assessments and item tracking. Bioinformatics. 2010;26(12):1572-3.

15. Habernau Mena A, Pozo VD, Abejón FF, Rodríguez Vidigal P, Bobadilla, González. Role of periostin in uncontrolled asthma in children (dado study). J Investigat Allergol Clin Immunol. 2017;27(5):291-8.

16. Troy NM, Hollams EM, Holt PG, Bosco A. Differential gene network analysis for the identification of asthma-associated therapeutic targets in allergen-specific t-helper memory responses. BMC medical genomics 2016; 9 (9.

17. Bai S, Hua L, Wang $X$, et al. Association of a 4-locus gene model including il 13 , il4, fcer $1 \mathrm{~b}$, and adrb2 with the asthma predictive index and atopy in chinese han children. J Investigat Allergol Clin Immunol. 2018;28(6):407-134.

18. Chlewicki LK, Velikovsky CA, Balakrishnan V, et al. Molecular basis of the dual functions of 2b4 (cd244). Journal of immunology (Baltimore, Md: 1950) 2008; 180(12):8159-67.

19. Gangwar RS, Minai-Fleminger Y, Seaf M, et al. Cd48 on blood leukocytes and in serum of asthma patients varies with severity. Allergy. 2017;72(6):888-95. 
20. Yoneyama M, Kikuchi M, Natsukawa $T$, et al. The rna helicase rig-i has an essential function in double-stranded rna-induced innate antiviral responses. Nature immunology. 2004;5(7):730-7.

21. Kim HK, Lee GH, Bhattarai KR, et al. Pi3kס contributes to er stress-associated asthma through erredox disturbances: The involvement of the ridd-rig-i-nf-kb axis. Exp Mol Med. 2018;50(2):e444.

22. Pang LL, Yuan XH, Shao CS, et al. The suppression of innate immune response by human rhinovirus c. Biochem Biophys Res Commun. 2017;490(1):22-8.

23. Menzel M, Ramu S, Calvén J, et al. Oxidative stress attenuates tlr3 responsiveness and impairs antiviral mechanisms in bronchial epithelial cells from copd and asthma patients. Frontiers in immunology 2019; 10(2765.

24. Shi K, Ge MN, Chen XQ. Coordinated DNA methylation and gene expression data for identification of the critical genes associated with childhood atopic asthma. Journal of computational biology: a journal of computational molecular cell biology. 2020;27(1):109-20.

25. Mackay F, Silveira PA, Brink R. B cells and the baff/april axis: Fast-forward on autoimmunity and signaling. Curr Opin Immunol. 2007;19(3):327-36.

26. Kumar R, Williams LK, Kato A, et al. Genetic variation in b cell-activating factor of the tnf family (baff) and asthma exacerbations among african american subjects. J Allergy Clin Immunol. 2012;130(4):996-.e6.

27. Yoshimura T, Oppenheim JJ. Chemokine-like receptor 1 (cmklr1) and chemokine (c-c motif) receptorlike 2 (ccrl2); two multifunctional receptors with unusual properties. Experimental cell research. 2011;317(5):674-84.

28. Haworth $\mathrm{O}$, Cernadas M, Levy BD. Nk cells are effectors for resolvin e1 in the timely resolution of allergic airway inflammation. Journal of immunology (Baltimore, Md: 1950) 2011; 186(11):6129-35.

29. Duan HJ, Li XY, Liu C, Deng XL. Chemokine-like factor-like marvel transmembrane domain-containing family in autoimmune diseases. Chin Med J. 2020;133(8):951-8.

30. Wang Z, Song K, Zhao W, Zhao Z. Dendritic cells in tumor microenvironment promoted the neuropathic pain via paracrine inflammatory and growth factors. Bioengineered. 2020;11(1):661-78.

31. Sun X, Liu X, Xia M, et al. Multicellular gene network analysis identifies a macrophage-related gene signature predictive of therapeutic response and prognosis of gliomas. Journal of translational medicine. 2019;17(1):159.

32. Tuipulotu DE, Netzler NE, Lun JH, et al. Rna sequencing of murine norovirus-infected cells reveals transcriptional alteration of genes important to viral recognition and antigen presentation. Frontiers in immunology $2017 ; 8(959$.

\section{Figures}



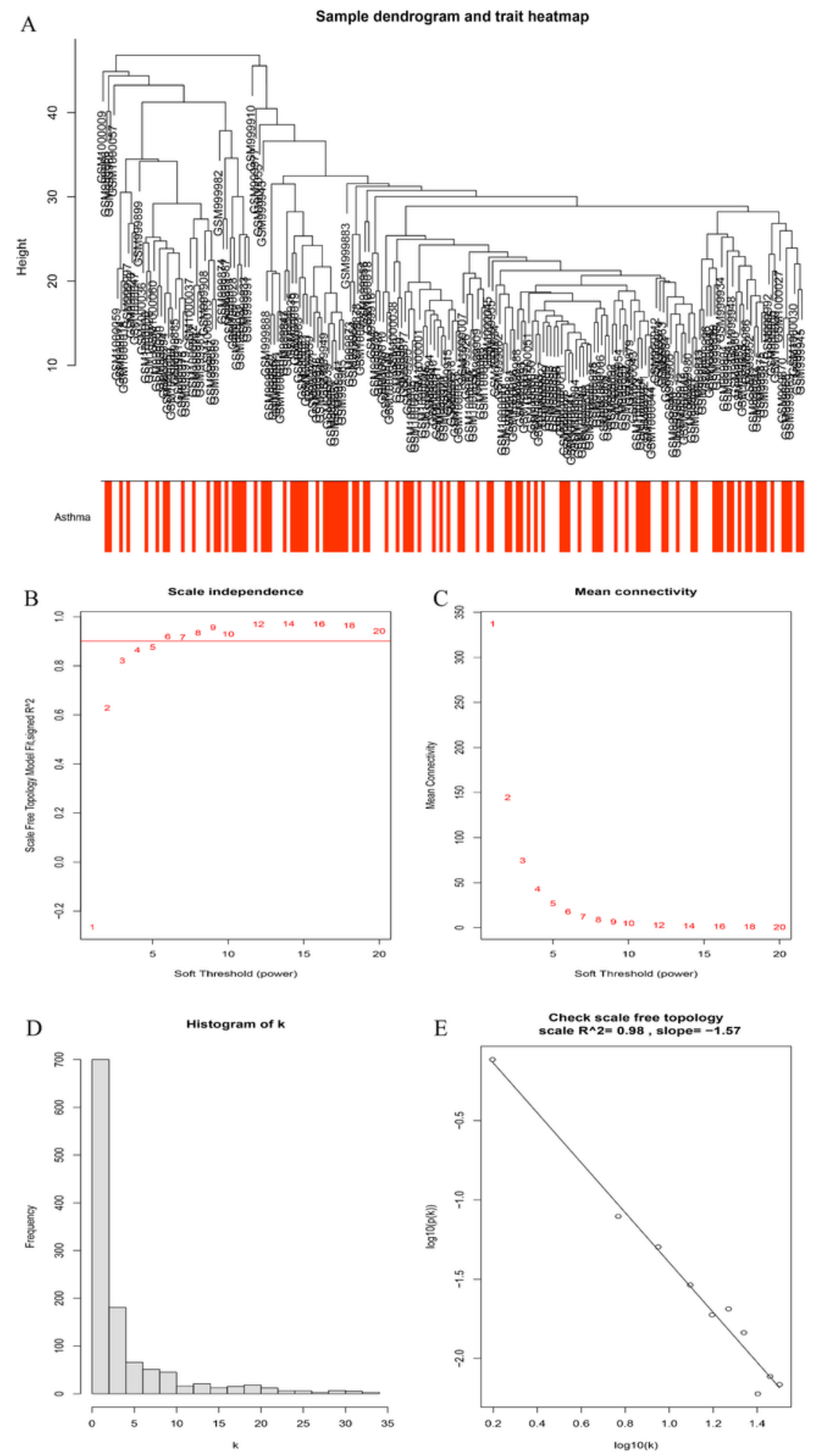

\section{Figure 1}

Clustering dendrogram of 97 non-asthma and 97 asthma patients from the GSE40732 dataset. (A) Screening for an appropriate $\beta$ in co-expression network. (B) Scale-free topology model fit index analysis based on various $\beta$ s. (C) Mean connectivity analysis based on various $\beta$ s. (D) Histogram of connectivity distribution for $\beta=4$. (E) Scale-free topology for $\beta=4$. 
A

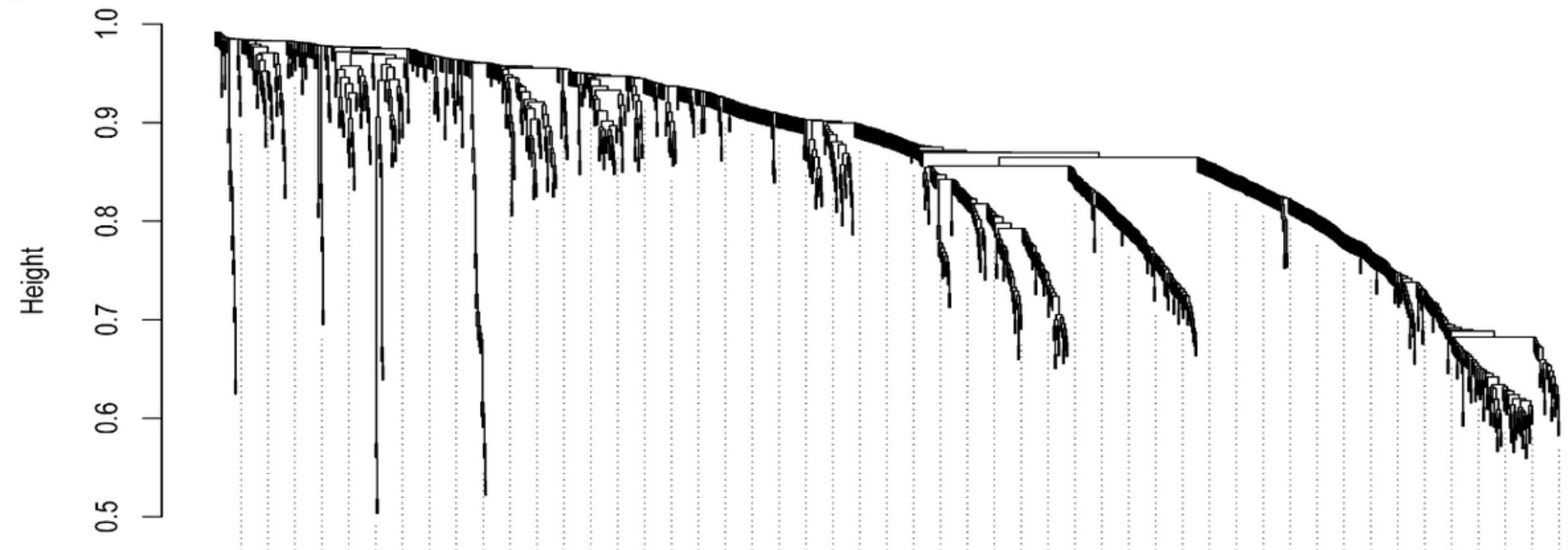

Dynamic Tree Cut

Merged dynamic
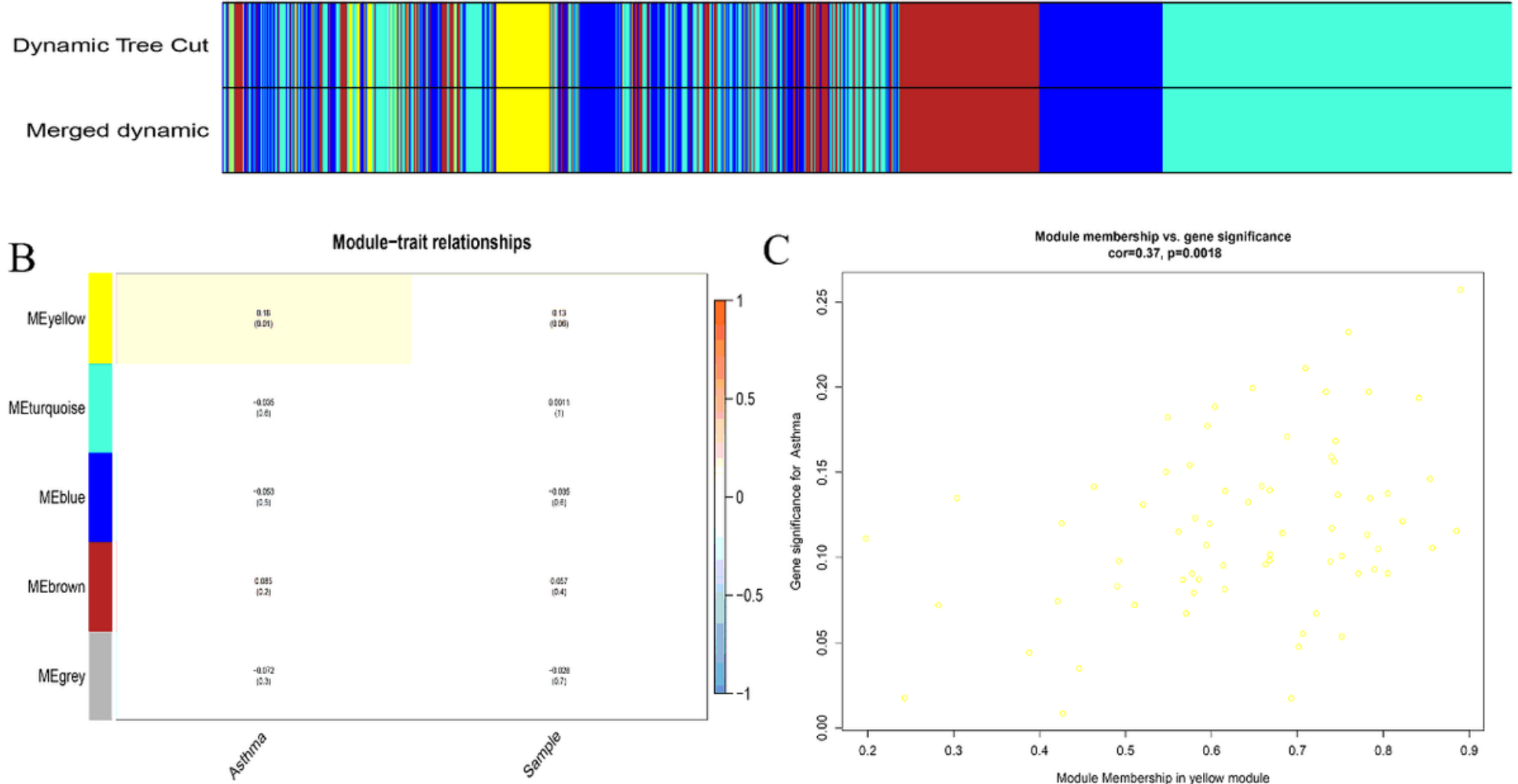

C

Module membership vs. gene significance

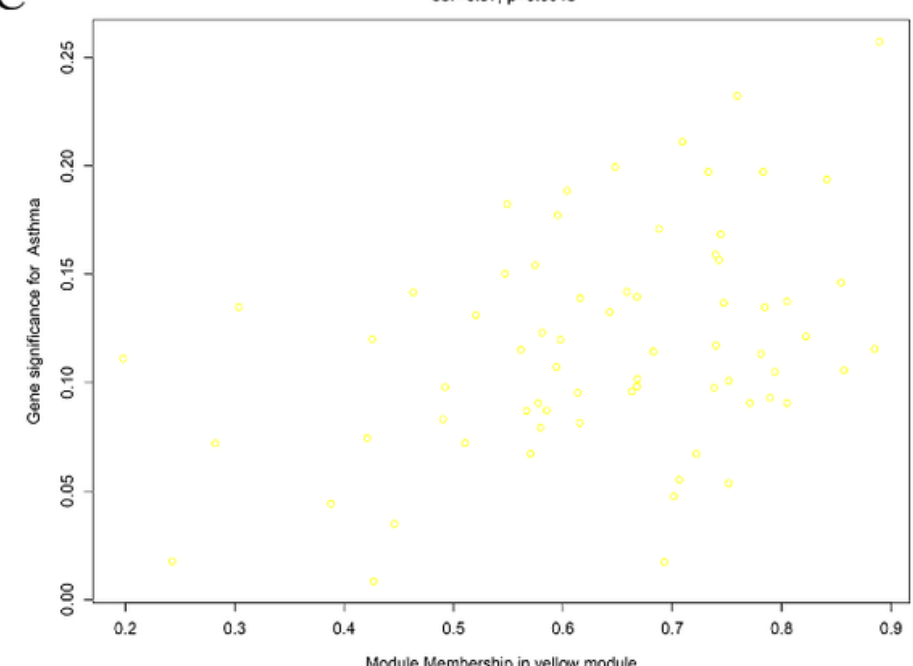

Figure 2

Identification of yellow modules related to the occurrence of childhood asthma. (A) Identification of five gene modules based on the dynamic shear tree. (B) The yellow module was closely correlated with the occurrence of childhood asthma, according to the module-trait relationships. (C) Gene scatter diagram in the yellow module. 


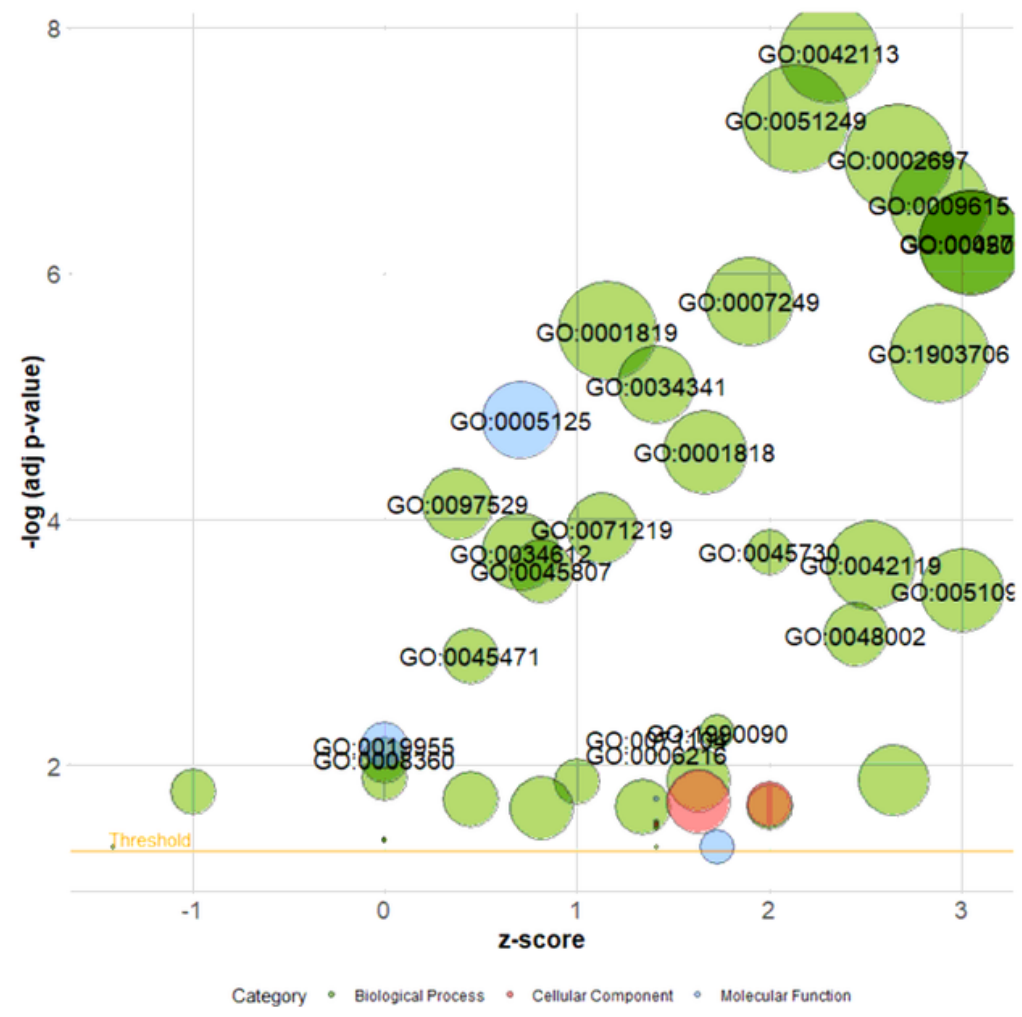

\begin{tabular}{|c|c|} 
ID & Description \\
\hline GO:0042113 & B cell activation \\
GO:0051249 & regulation of lymphocyte activation \\
GO:0002697 & regulation of immune effector process \\
GO:0009615 & response to virus \\
GO:0002768 & immune response-regulating cell surface receptor signaling pathway \\
GO:0045088 & regulation of innate immune response \\
GO:0007249 & I-kappaB kinase/NF-kappaB signaling \\
GO:0001819 & positive regulation of cytokine production \\
GO:1903706 & regulation of hemopoiesis \\
GO:0034341 & response to interferon-gamma \\
GO:0001818 & negative regulation of cytokine production \\
GO:0097529 & myeloid leukocyte migration \\
GO:0071219 & cellular response to molecule of bacterial origin \\
GO:0045730 & respiratory burst \\
GO:0034612 & response to tumor necrosis factor \\
GO:0042119 & neutrophil activation \\
GO:0045807 & positive regulation of endocytosis \\
GO:0051090 & regulation of DNA-binding transcription factor activity \\
GO:0048002 & antigen processing and presentation of peptide antigen \\
GO:0045471 & response to ethanol \\
GO:1990090 & cellular response to nerve growth factor stimulus \\
GO:0071104 & response to interleukin-9 \\
GO:0006216 & cytidine catabolic process \\
GO:0008360 & regulation of cell shape \\
GO:0005125 & cytokine activity \\
GO:0019955 & \\
&
\end{tabular}

Figure 3

Gene ontology (GO) analysis that explores the potential mechanism underlying the effect of significant immune-related genes on the occurrence and development of childhood asthma, using 'clusterProfiler' package in R software. 
A

Reverse cumulative distribution of |residual|

model $-n-\ldots . m-$ sm

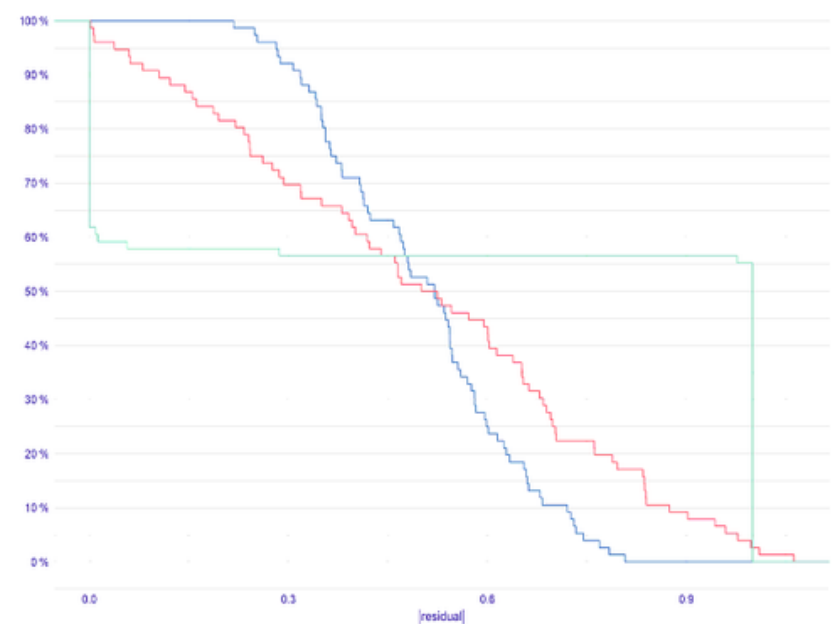

$\mathrm{C}$

Top 30 - variable importance
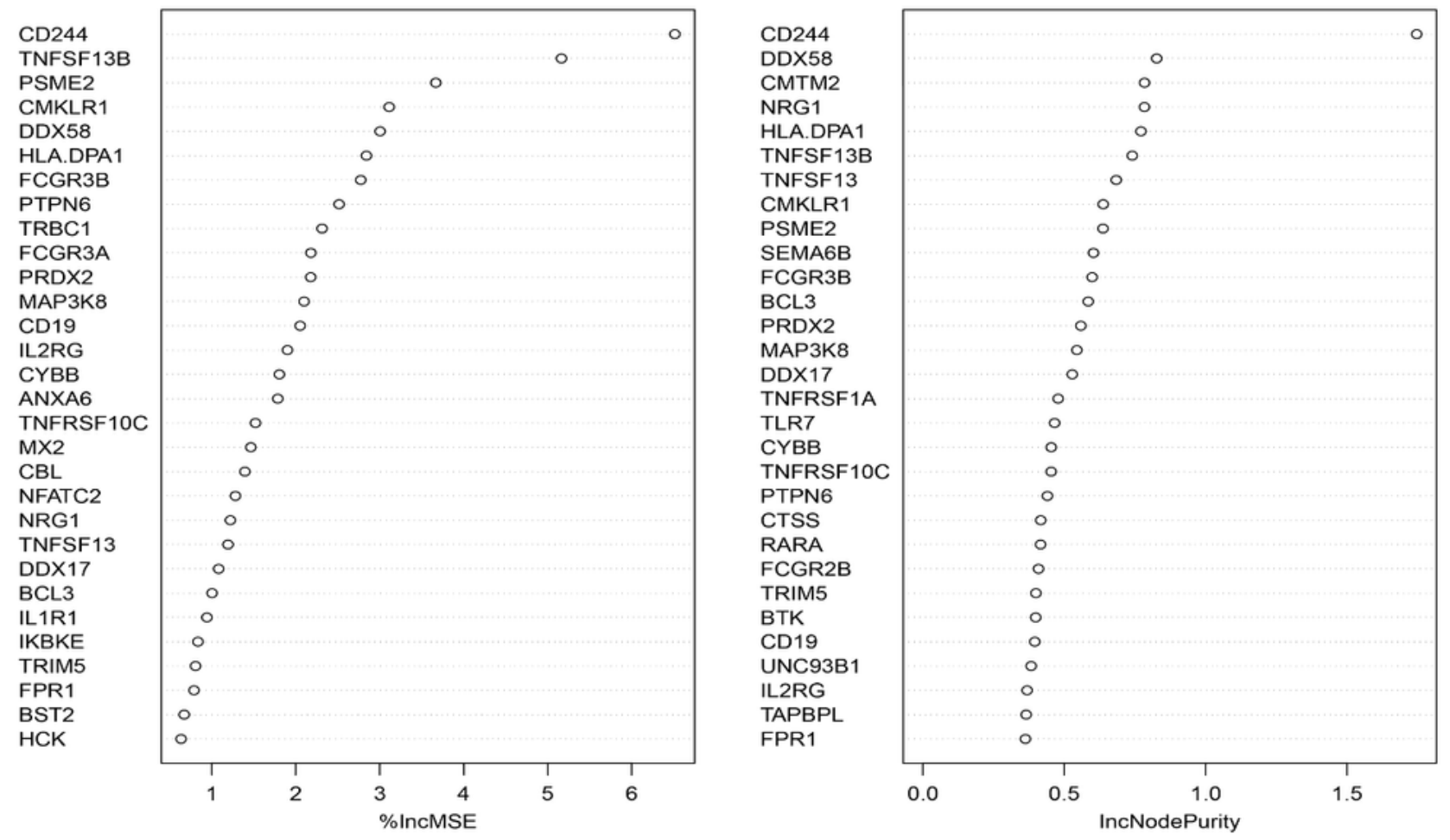

Figure 4

Random forest model construction. (A) Cumulative residual distribution map of the RF, GLM, and SVM models. (B) Histogram of the residual distribution of the RF, GLM, and SVM models. (C) The importance of the top 30 immune-related genes based on the RF model. 
A

Points

CD244

CMKLR1

CMTM2

DD $\times 58$

HLA.DPA1

NRG1

PSME2

SEMA6B

TNFSF13

TNFSF13B

Total Points

asthma

B

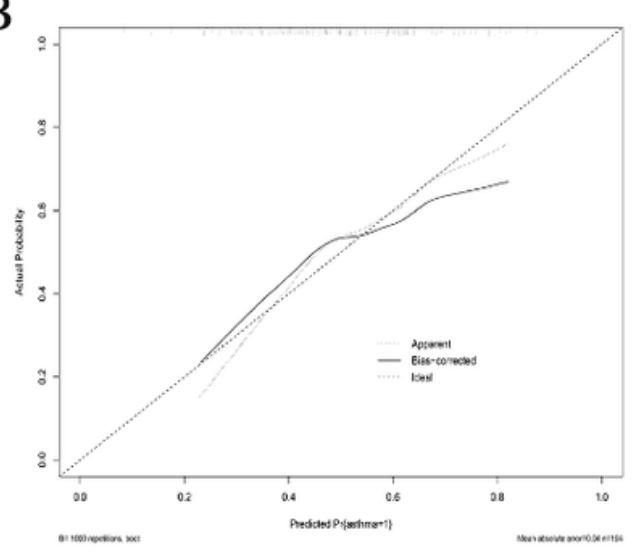

$\dot{6}$
ㅇ 10

o 20

30

40

50

60

70

80

90

\begin{tabular}{llllllll}
\hline 8 & 8.5 & 9 & 10 & 10.5 & 11.5 & 11 & 10
\end{tabular}

$$
10 \begin{array}{llllll}
10 & 8 & 7 & 6
\end{array}
$$

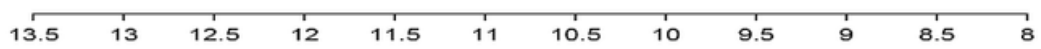

$\begin{array}{llllllllll}9.5 & 9 & 8.5 & 8 & 7.5 & 7 & 6.5 & 6 & 5.5 & 5\end{array}$

\begin{tabular}{rrrrr}
\hline $5^{\prime}$ & 14.4 & 13.8 & 13.2 & 12.6
\end{tabular}

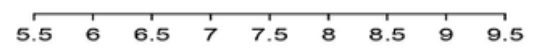

\begin{tabular}{ll|l|l|l}
\hline 10 & 11 & 12 & 13 & 14
\end{tabular}

$\begin{array}{llllllll}14 & 13 & 12 & 11 & 10 & 9 & 8 & 7\end{array}$

$8.510 \quad 12 \quad 14$

62060
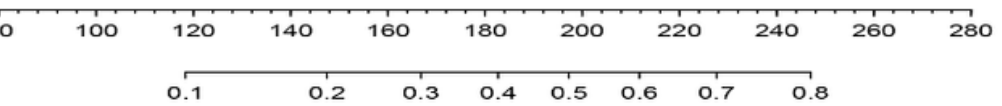

C

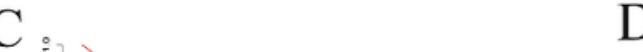

D
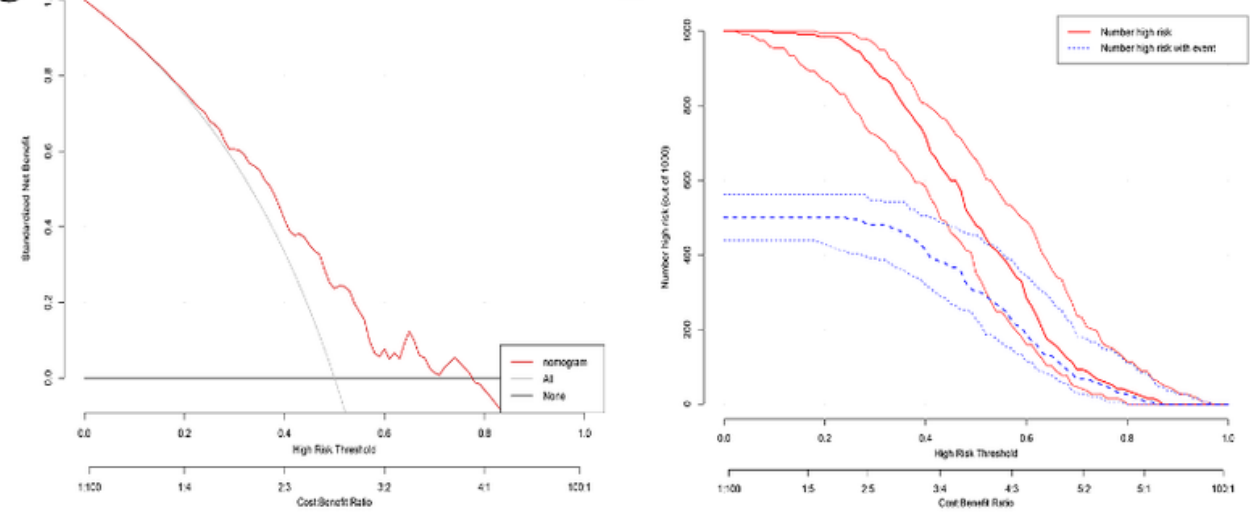

Figure 5

Construction of the nomogram model. (A) Construction of the nomogram model based on the top 10 immune-related genes. (B) Predictivity of the nomogram model as revealed by the calibration curve. (C) Decisions based on the nomogram model may benefit childhood asthma patients. (D) The clinical impact of the nomogram model as assessed by the clinical impact curve. 
A

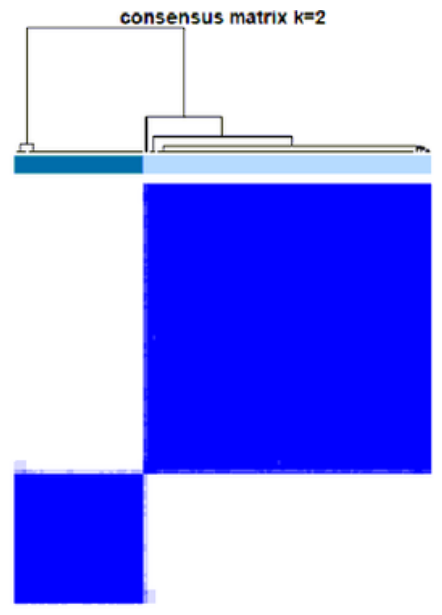

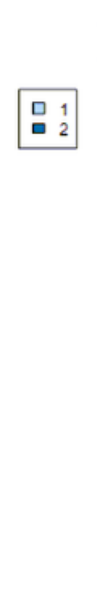

B

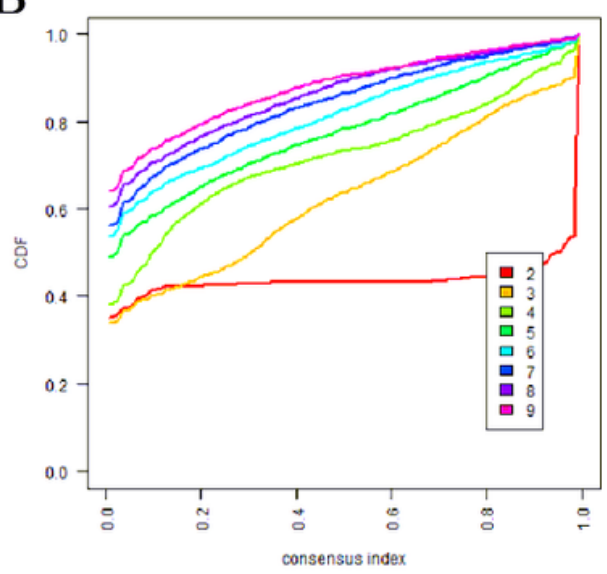

tracking plot

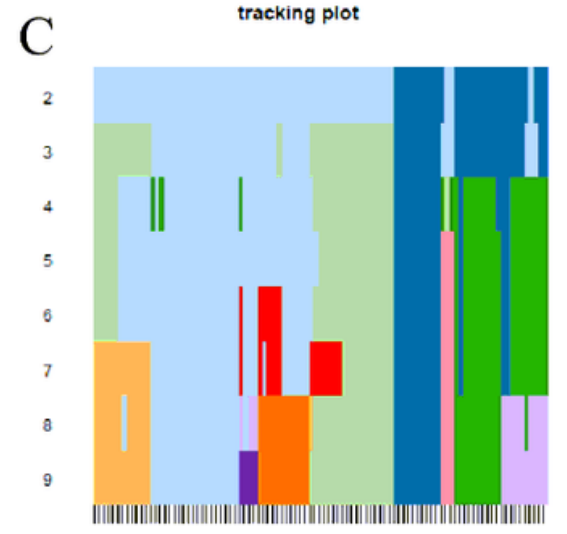

samples

Figure 6

Identification of two molecular subtypes of childhood asthma from the training dataset. (A) The matrix heat map was clearly separated when $k=2$. (B) The CDF reaches an approximate maximum value and the results of clustering analysis were the most reliable when $k=2$. (C) Tracking plot from $k=2$ to 9 . The black stripe at the bottom represents the sample, showing the classification of the sample when different values of $k$ are considered; the blocks of different colors represent different classifications. 
A GSE40732
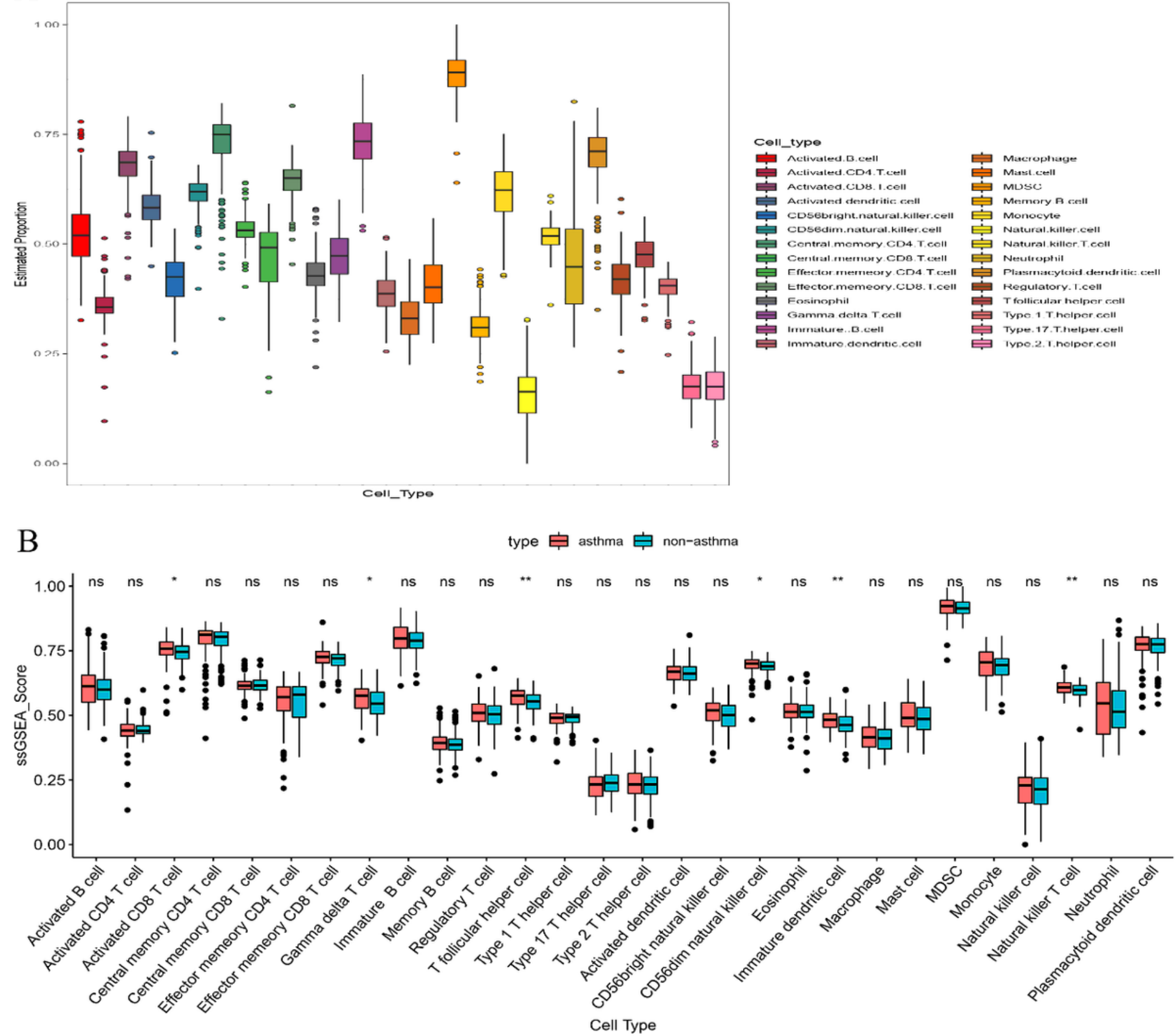

Figure 7

Single sample gene set enrichment analysis. (A) The abundance of 28 infiltrating immune cell types in childhood asthma patients from the training dataset. (B) Differential immune cell infiltration between non-asthma and asthma patients in the training dataset. 

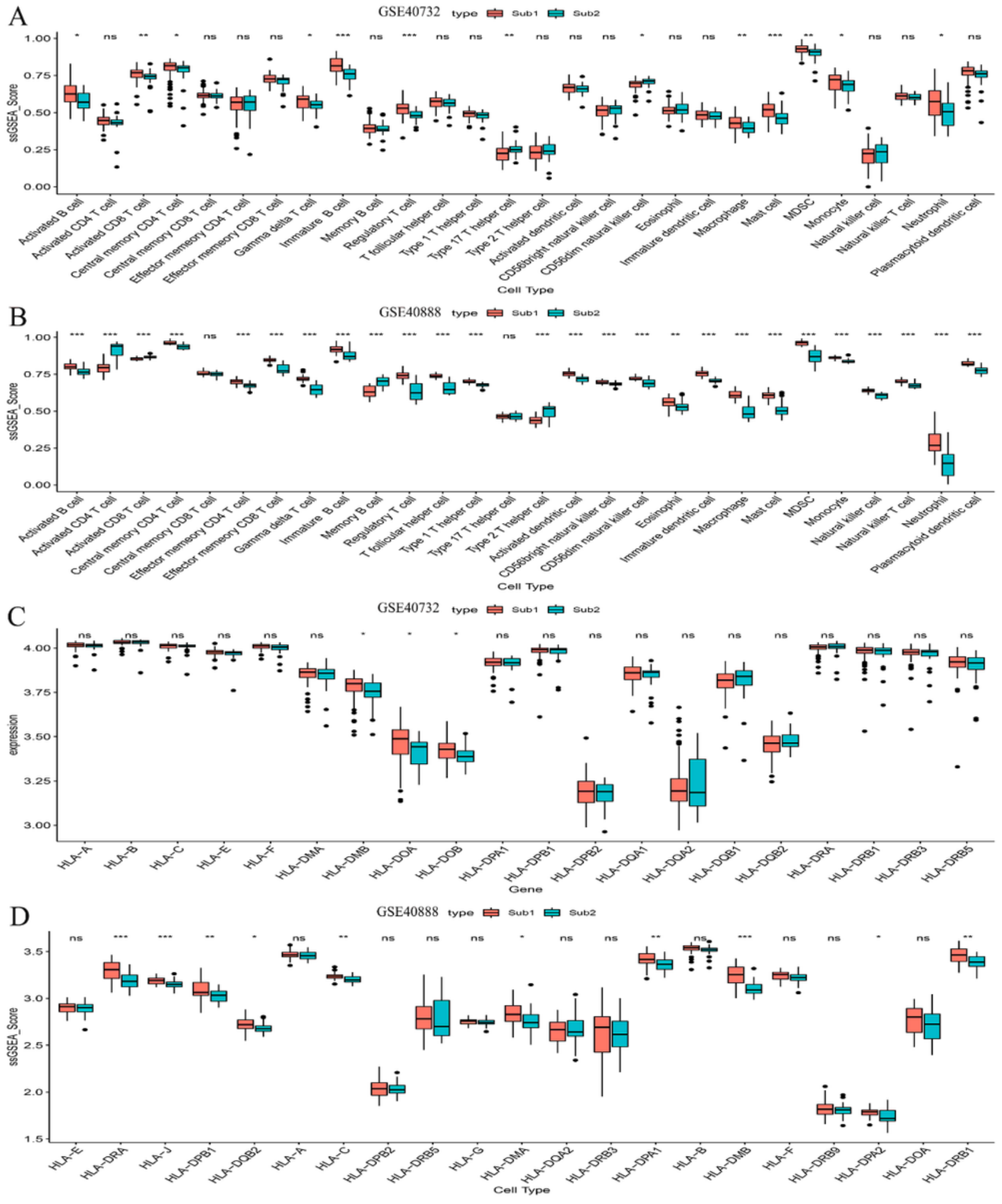

\section{Figure 8}

Biological function analysis of the two molecular subtypes. (A) Differential immune cell infiltration between the two molecular subtypes in the training dataset. (B) Differential immune cell infiltration between the two molecular subtypes in the testing dataset. (C) Comparison of the expression of human leucocyte antigen (HLA) genes between the two molecular subtypes in the training dataset. (D) 
Comparison of the expression levels of the HLA genes between the two molecular subtypes in the testing dataset.

\section{Supplementary Files}

This is a list of supplementary files associated with this preprint. Click to download.

- Supplementarymaterials.docx 